\title{
Structural blue coating based on glaze painting
}

\section{Bleu structurel d'origine non pigmentaire obtenu par la méthode des glacis en peinture}

\author{
Anne Goyer ${ }^{1}$, Amina Bensalah-Ledoux², Davy Carole ${ }^{3}$, Cécile Le Luyer², \\ Tiphaine Blanchard ${ }^{2,3}$, Isabelle Merdrignac ${ }^{4}$, Isabelle Guibard ${ }^{4}$, Anne Pillonnet $^{2^{*}}$ \\ ${ }^{1}$ Artist, Avignon, France \\ ${ }^{2}$ Institut Lumière Matière UMR 5306 CNRS - Université Lyon1, Université de Lyon, France, \\ anne.pillonnet@univ-lyon1.fr \\ ${ }^{3}$ Laboratoire Multimatériaux et Interfaces UMR 5615 CNRS - Université Lyon1, Université de Lyon, France \\ ${ }^{4}$ IFP Energies nouvelles - Solaize, France
}

ABSTRACT. An art/science collaboration has enabled a contemporary artist to re-discover an age-old technique used by glaze-masters such as Leonardo da Vinci. The process uses dark bitumen, white mineral particles, a brush, and knowledge of drying and laying time to produce intense, structural, blue hues that are mainly due to Rayleigh light scattering. Although these blue hues are mentioned in ancient studio manuscripts, very few are found in actual easel painting masterpieces.

KEYWORDS. Art/Science, Glaze oil painting, Structural coloration, Nanoparticles, Rayleigh light scattering.

\section{Introduction}

For centuries, the secrets of famous Renaissance painters have stirred the curiosity of ardent art lovers. Some of their knowledge has survived until the present by being passed down through generations of art-restorers in transcripts of ancient texts ${ }^{[1,2]}$, complemented by the physical and chemical analysis of master paintings ${ }^{[3-6]}$. These advances in knowledge have economic consequences for the art market and can sometimes provide an inspiration to the science of material, as we propose here. The manuscript written by Leonardo da Vinci and published in 1651 by Francesco Melzi as part of "Trattato della pittura" is one of several to mention a particular type of blue ("azzurro") that was produced by depositing very fine lead white pigment onto a black background ${ }^{[7]}$. Da Vinci compared this colour to the one produced by smoke in a chimney flue and to the hues observed in the sky. These unpigmented structural colours are created by light scattering on small particles, an effect which was explained in the late 19th century by Tundall and Rayleigh ${ }^{[8]}$ and is now commonly referred to as Rayleigh scattering. This particular type of blue has also been observed in porcelain and ceramics enamels ${ }^{[9,10]}$. Their nanoscale structure consists of crystallites dispersed in a glass matrix that were fixed at high temperatures and have withstood the test of time allowing us to still appreciate this colour today. Over the past decades, unpigmented coloured coatings have received scientific attention, often inspired by the observation of bio-organisms ${ }^{[11-18]}$. This research has included the use of (i) controlled multi-layers to create planar constructive interference, (ii) mastered periodic structures named photonic crystals, (iii) quasi-amorphous structures that are ordered over short ranges but amorphous over long ranges. The quasi-amorphous structures consist of closely packed air cavities in $\beta$-keratin and cause the non-iridescence blue colour of the plum-throated cotinga's (Cotinga maynana), a bright turquoise bird of Peru and Ecuador $^{[17]}$ or of human blue eyes ${ }^{[18]}$, for instance. To produce this phenomenon, a self-assembly of mono-dispersed particles are typically used ${ }^{[13-16]}$. Colours from blue to magenta in this way can be attributed to the constructive interference of scattered light from quasi-amorphous structures. The blue created by Rayleigh scattering superimposes on colours produced by interference effect ${ }^{[13]}$. In 
this paper, we report a novel approach to coloration based on the glaze oil painting technique to generate a structural blue coating using quasi-amorphous structures of poly-dispersed particles.

Artists have used oil paints since the $8^{\text {th }}$ century but they only became common in the early $15^{\text {th }}$ century when Flemish artists such as van Eyck created exquisite works from repeated transparent layered colours painting these glazes over the opaque oil layers ${ }^{[4,19]}$. Since oil dries at a slower rate and more evenly than tempera, the artist had more time to correct and extend their work which gave a greater transparency to the glaze which in turn increased hue saturation.

Here, this layering approach has been re-created through a collaboration between art and science. Initially, the contemporary artist, Anne Goyer has harnessed a bitumen medium in drawing design ${ }^{[20,21]}$. She has produced glazes that combine brown natural bitumen, manually crushed white mineral particles, translucent binders, and solvent to produce a shallow blue hue with very few ingredients and without the use of blue pigment. The scientific study of the interactions between light and matter that create the blue coloration have resulted in an optimized protocol. An intense blue colour can be created, in a reproducible way, without the use of blue pigments on paper, using a simple brush, mortar, as well as other natural and affordable raw materials. These contemporary glazes were obtained following a manual protocol and its structure and its thickness are comparable to the glazes used in ancient paintings as documented in the literature of that time.

\section{Structural blue design}

In a first attempt to produce structurally blue coloured eyes, the artist employed an intuitive approach to create an oil-based glaze (Figure 1A). This resulted in a blue tint was shallow and that could not be systematically reproduced. Through the collaboration with science, it became possible to establish a protocol that reliably reproduced the same type of blue that was also much more intense (Figure 1B). By analysing the artist's initial technique the scientists were able to identify the optical phenomena at the origin of the structural blue colour and suggest improvements to the methodology which were tested by the artist. This has resulted in the establishment of a new protocol that is based on the same original ingredients and that does not require any technological input (e.g., for particle size selection, particle coating in the lab).

The new optimized process to obtain a vivid blue structural colour involves three steps: (i) preparing the paper base; (ii)creating a dark background layer; and (iii) mounting the particles into a transparent matrix to form the glaze (Fig. 1C).

For the first step, the paper is soaked with varnish (Vernis de Retouche Surfin, Lefranc Bourgeois) to reduce the penetration of the pictorial layer into the paper. Unbleached Sennelier paper with a density of $80 \mathrm{~g} \mathrm{~m}^{-2}$ is used as the use of thicker paper yields less conclusive results. From weight measurements, we determined that about $70 \%$ of the varnish had evaporated after drying the varnished paper for 50 minutes at room temperature (Figure 2).

The second step is the creation of a dark background layer. This was achieved by applying five coats of bitumen with drying times of at least one hour between each coating to form a continuous and homogeneous layer. While the first four layers consist of bitumen diluted with rectified turpentine, the final layer consists of pure bitumen. Different oils and acrylic paint-based black background layers were tested but they did not lead to the obtaining of the blue hue..

The bitumen used in this study was sourced from the former natural hydrocarbon source at Puy de la Poix (Auvergne, France). This bitumen is a type of pitch that was created from the sediments that formed in Limagne Lake during the Oligocene (34 to $23 \mathrm{Ma}$ BCE). Bitumens are complex mixtures of hydrocarbon compounds with various chemical structures and molecular weights ${ }^{[22,23]}$. 
By comparing the elemental and molecular composition, density, viscosity and refractive index, of the bitumen from Puy de la Poix to the bitumen found in Judea, which was typically used in Renaissance period paintings ${ }^{[24,25]}$, we found that the percentage content of oxygen and sulphur in our bitumen was elevated, possibly due to oxidation (Table S1). In addition to these heteroatoms, we also found metals in trace amounts (Table S2), as well as high quantities of sodium, iron, calcium and sulphur, which are characteristic of the lacustrine origin of the natural source. Bitumen are typically described by classifying its components according to their polarity into four families: saturated compounds, aromatic compounds, resins, and asphaltenes (Table S3). For our bitumen, the colloidal instability index, i.e., the ratio between (asphaltene + saturated compounds) and (resins + aromatic compounds) was found to be 0.365 which is characteristic of bitumen of type 'sol'. Sol bitumens have both viscoelastic and viscous characteristics that resemble those of a Newtonian fluid $^{[23]}$. The viscoelastic properties explain the shiny and homogenous dark coating. Besides, the density d15/4 reachs $1060 \mathrm{~kg} \cdot \mathrm{m}^{-3}$ and the refractive index (at $70^{\circ} \mathrm{C}$ ) equals 1.558 at a wavelength of $\lambda=589 \mathrm{~nm}$. These values are higher than for Judea bitumen which has a density of $850 \mathrm{~kg} \cdot \mathrm{m}^{3}$ and a refractive index of 1.485 (Table S4). The layer of pure bitumen deposited on soaked paper has lost about $33 \%$ of its weight 50 minutes after deposition (Fig. 2). This corresponds to the weight percentage of the aromatic and saturated compounds which have a lower molecular weight and are thus more volatile (Table S3).

In the last step, the blue glaze is added to the previously described background layer applying 8 to 12 consecutive coatings. Note that only two coatings have been included in Figure 2B. Each coating is applied using a brush and consists of dry white mineral particles covered with a fine layer of spray-coated oil (charcoal and pastel fixative, Conté de Paris). The mineral particles are manually ground on a marble slab before deposition. The oil must be colourless, and transparent. While the oil on paper only requires a drying time of 50 minutes it needs to dry for an entire day before the next coating can be applied in order to obtain the blue coloration. The white mineral particles were PW6 or PW5 Rembrandt soft pastel which are mainly composed of lithopone (zinc sulphide (ZnS) and barium sulphate $\left(\mathrm{BaSO}_{4}\right)$ ) and a small amount of rutile $\left(\mathrm{TiO}_{2}\right)$ as determined from X-ray diffraction and X-fluorescence spectroscopy (Figure S1).

\section{Light and matter interactions}

The blue hue that appears in the drawings in Fig. (1) does not contain any blue pigments. In order to explain the origin of this blue colour and to identify the role of each component of the multilayer, the individual raw materials, i.e., bitumen, rectified turpentine, oil, and mineral particles were deposited individually on the same type of paper and their optical reflectance was analyzed (Figure 3A). The dark background layer of bitumen predominantly absorbs the shorter wavelengths which results in a warm shade of dark brown.

The layer of rectified turpentine appears in a light yellow when in contact with air as evidenced by the wide absorption band below 500nm (Fig. 3A). The oil layer remains even after drying.

Assuming the virgin paper has a reflectance of $100 \%$, the reflectance of the mineral particles is greater than $100 \%$ throughout the visible range with higher intensities at shorter wavelengths yielding a bright white appearance. This is caused by photoluminescence at $450 \mathrm{~nm}$ from an excitation wavelength of 350nm (Figure 3B). This blue luminescence is due to rare earth impurities naturally present in barite ${ }^{[26]}$ and to the presence of zinc sulphide ${ }^{[27]}$, both components of lithopone. These materials are often used as optical brighteners ${ }^{[28]}$. Laser diffraction spectroscopy and scanning electron microscopy (SEM) could show that the manual grinding of these white particles on a marble slab yields three different size groups of particles: nanoparticles close to $100 \mathrm{~nm}$ in diameter and microparticles of about $8 \mu \mathrm{m}$ and $250 \mu \mathrm{m}$ (Figure 3C). The nanoparticles are smaller than 
visible wavelengths and could generate Rayleigh scattering (favouring shorter blue wavelengths), while the microparticles generate Mie scattering which is wavelength independent (giving rise to white aspect) ${ }^{[8-12]}$.

The reflectance spectra of the three multilayers show how the colour evolved from a grey blue when using the artist's intuitive approach to a more vivid blue when using the systematic sciencebased approach (Fig. 1C). While the intuitive approach yielded a reflectance at the critical 430nm wavelength, the more systematic science-based approach increases this reflectance. The range of blue shades accessible with this process has been evaluated in CIELAB colour space ${ }^{[29]}$ (Figure S2) yielding a lightness parameter $\mathrm{L}^{*}\left(0>\mathrm{L}^{*}<100\right)$ between 46 and 83 . The $\mathrm{a}^{*}$ parameter covers the red-green components $\left(-128\right.$ (green) $<\mathrm{a}^{*}<127$ (red)) and was found to differ only slightly from zero with values between -1 and +3 indicating the near absence of any red or green components. The $b^{*}$ parameter covers the blue-yellow components and was always negative with values between -5 and -22 which indicates blue coloration.

Illuminating the same painting with UV light $(354 \mathrm{~nm})$ reveals some large purple areas, such as the left cheek and nose, which correspond to the presence of mineral particles (Fig. 3D). The darker parts correspond to the concentrated bitumen layer and the absence of mineral particles and the clear areas correspond to the virgin or oil soaked paper. While the eyes appear relatively similar under natural sun light, they seem very different when illuminated with UV light. The intuitive realization of the blue (cf. Fig. 1A) did not always follow the same protocol. If the blue colour observed in the painting was only due the mineral particles, all areas that appear purple under UV illumination would appear blue under sunlight. However, the blue only appears in those areas where the complex glaze protocol has been used, i.e., for the pupils of the eyes (cf. Fig. 3).

SEM images show cross section of the layered structure (Figure 4). While on the right side of Fig. 4a the paper is only covered by the bitumen background layer, on the left, the paper is covered by both the background and glaze layers. The paper substrate consists of an assembly of paper fibres and is about $120 \mu \mathrm{m}$ thick (based on manufacturer-specified weight of $80 \mathrm{~g} \mathrm{~m}^{-2}$ ) which corresponds to a density of $667 \mathrm{~kg} \mathrm{~m}^{-3}$. The paper is thus relatively porous which highlights the role played by the paper varnish and soaking process to prevent bitumen diffusion. The thickness of the bitumen background layer is between 10 and $13 \mu \mathrm{m}$. The glaze layer with embedded mineral particles consists of 10 smaller layers with a combined thickness of around $33 \mu \mathrm{m}$. The degree of magnification of the glaze layer is sufficiently large allowing the sizes of the mineral particles to be estimated. Comparing them to the original size distribution of the particles after manual grinding (Figure 3C) only the nanoparticles appear visible. The coating expertise of the artist enables the selection of the nanoparticles to promote the structural blue colour by scattering. The presence of nanoparticles is key to obtain the structural blue. If the bigger particles were also present in the glaze layer, the multi-layered structure would appear white. The thickness of our glaze layer is similar to the thickness of glaze layers in old paintings, particularly in those that date from the period where the so-called sfumato technique was first developed ${ }^{[4]}$.

\section{Discussion}

This study demonstrated that the pigment-free blue hue is caused by Rayleigh scattering by highly polydispersed mineral particles incorporated into a transparent matrix with a contribution of near UV induced fluorescence. There is no constructive interferences of the scattered light due to the polydisperse particle sizes. Previous work on unpigmented structural coloured coatings based on monodisperse randomly distributed particles found that the colour was controlled by the particles size ${ }^{[13,15]}$ or by the distance between particles ${ }^{[14]}$. The colour observed with monodisperse particle is mainly due to constructive interferences of scattered light with a blue Rayleigh scattering 
contribution $^{[13]}$ which makes it difficult to obtain a red colour from these kind of structures. The intense blue colour we observed in our glaze is more like the blue of monolithic aerogels doped with polydisperse polystyrene particles with diameters of roughly $50-100 \mathrm{~nm}^{[30]}$ or of hydrothermal lake water naturally doped with colloidal silica particles of $100-450 \mathrm{~nm}$ in size ${ }^{[31]}$. Usually, this blue is attributed to Rayleigh or Tundall scattering, depending on the size of the particles, and produces the blue of the sky ${ }^{[8,32,33]}$ and the bluish smoke that emanates from chimneys ${ }^{[7,8]}$. We could show that a simple brush only selects the smaller nanoparticles that are important for the Rayleigh scattering and thus the blue colour. Moreover, depositing the glaze layers directly on a dark bitumen background made the blue appear more intense. This is a well known phenomenon and has been studied by Fudouzi et al. ${ }^{[14]}$ who prepared and studied photonic papers that consisted of nanoparticle arrays dispersed in polymer films. These photonic papers have a pale appearance on white background and become coloured on a black background. More recently, Ge et al, ${ }^{[13]}$ described the same phenomenon for quasi-amorphous arrays of silica nanoparticles spray-coated onto glass. Furthermore, the high refractive index of bitumen $(1.558$ at $589 \mathrm{~nm})$ and its smooth surface create a high specular reflectance at the glaze/bitumen interface. This reduces the amount of angle independent scattering allowing the blue Rayleigh scattering to appear less polluted by other colours except at the specular reflection angle.

From an artistic point of view, this study could demonstrate that it is possible to create a blue coloured paint that does not contain any blue pigments, thus creating a direct reference to the glaze technique invented in the $15^{\text {th }}$ century.

While bitumen from natural sources has been used since antiquity in paintings ${ }^{[24,25]}$, its use on canvas or wood has generated significant aging-related problems such as cracks, discolorations, or colour shifts due to chemical reactions between the non-dried bitumen and pigments, as can be observed in Géricault's "Le Radeau de la Méduse". Due to natural oxidation on contact with the air, the bitumen from Puy de la Poix that was used in this study is stable in air and at room temperature in the absence of other reactants. We also did not mix the bitumen with mineral particles and by depositing it on thin porous paper the paper fibres quickly absorb the bitumen and facilitate drying which should make paintings more durable.

While paintings produced since the $19^{\text {th }}$ century use white lithopone and titanium oxide particles ${ }^{[28]}$, earlier works typically used white lead, sometimes mixed with chalk ${ }^{[1]}$. According to Gonzales et al. ${ }^{[3]}$, the analyses of the white lead pigments extracted from a selection of Renaissance easel paintings yielded particle sizes below 100nm, i.e., consistent with Rayleigh scattering conditions. The authors proposed levigation post-synthesis, the Dutch stacking method, and aqueous synthesis as potential fabrication processes.

The last important ingredient is the binder which must be transparent and colourless. During the Renaissance period, typical binders were linseed oil or egg yolk, which both have a yellow colouring that increases over time. The Van Eyck Brothers found a method to whiten the linseed oil by exposing it to UV illumination in an inert atmosphere. Paintings were also covered by layers of varnish with similar yellowing properties. Thus, even if a pigment-free blue would have been used at the time, it would be difficult to observe today.

Except for the manuscripts written by Leonardo da Vinci ${ }^{[7]}$ the blue hue remained unnoticed in master paintings. According to DeBehault ${ }^{[2]}$, Rubens exploited blue-hued scattering in his paintings, often in combination with another pigment to produce violet or green tints. Several references about optical phenomena are in the treatise he wrote together with physicist Franciscus Aguilonius ${ }^{[2]}$. The observed discoloration in Rubens paintings due to aging is probably a consequence of the oil and/or varnish yellowing and to the increase of the refractive index of the binding medium that stiffens 
over time. Typically, the blue coloured glazes in easel paintings used ordinary blue pigments such as lapis lazuli, azurite, iron, or cobalt blue ${ }^{[5,6]}$.

\section{Conclusion}

This study showed how thanks to the mastery of the technical gesture, basic ingredients such as bitumen, crushed white mineral particles, and binders can be ingeniously combined to create a pigment-free vivid blue, akin to the ones used by Renaissance masters. We created a pigment-free blue by employing Rayleigh scattering and photoluminescence on nanoparticles embedded in an otherwise transparent glaze. The term consecrated by the painters is 'opalescence', it is connected to all phenomena of light scattering caused by 'velatura' or clear glazes on a dark background. Science provided the necessary explanation of the light-matter interactions that give rise to the blue colour and allowed the process to be optimized and become reproducible. Ways how not to proceed have been identified: (i) the use of solvent and oil that have even a weak absorption in the short wavelength range obliterates the blue; (ii) an inhomogeneous or cracked background layer causes diffuse reflections of the long wavelengths; (iii) failure to allow the necessary drying time between adding the different layers results in poor mineral particle dispersion in the glaze.

This method to create colours from light scattering and photoluminescence provides an alternative to the use of chemical pigments. The method is simple to implement, on various support at room temperature, such as paper. Other colours could be produced using similar techniques and quasi-disordered networks of monodisperse particles. These results raise questions about the practices used by the ancient masters before the chemical production of blue pigments became affordable. This work demonstrates the strength of cross- and multi-disciplinary research to elucidate and promote new practices and knowledge.

\section{References}

[1] S. Bucklow, in The Matter of Art: Materials, Practices, Cultural Logics, c. 1250-1750, (Eds: C. Anderson, A. Dunlop, P.H. Smith), Manchester University Press, UK, 2015, Ch. 6.

[2] S. De Behault, in The Create2010 Conference Proc., (Eds: G. Simone, J.Y. Hardeberg, I. Farup, A. Davis, C. Parraman), 2010, p. 86

[3] V. Gonzalez, T. Calligaro, G. Wallez, M. Eveno, K. Toussaint, M. Menu, Microchemical Journal 2016, 125, 43.

[4] L. De Viguerie, P ; Walter, E. Laval, B. Mottin, V. Armonado Solé, Angew. Chem. 2010, 122, 6261.

[5] N. Grassi, A. Migliori, P.A. Mando, H. Calvo del Castillo, Nucl. Instrum. Methods Phys. Res., Sect. B, 2004, 219220,48 .

[6] T.L. Dawson, Color. Technol., 2007, 123, 281.

[7] L. De Vinci, Trattato della pittura, 1478-1518, compiled by F. Melzi in 1651, translated by A. Chastel, BergerLevrault Ed., Paris, 1987.

[8] J.W.S. Rayleigh, Phil. Mag. S., 1899, 5, 47, 375.

[9] Y. Yang, M. Feng, X. Ling, Z. Mao, C. Wang, X. Sun, M. Guo, J. Archaeol. Sc., 2005, 32, 301

[10] P. Shi, F. Wang, J. Zhu, B. Zhang, T. Zhao, Y. Wang, Y. Qin, Ceramics International , 2017, 43, 2943

[11] S. Kinoshita, S. Yoshioka, J. Miyazaki, Rep. Prog. Phys., 2008, 71, 076401.

[12] P. Vukusic, J.R. Sambles, Nature, 2003, 424, 852.

[13] D. Ge, L. Yang, S. Yang, G. Wu, J. Mater. Chem. C, 2014, 2, 4395.

[14] H. Fudouzi, Y. Xia, Langmuir, 2003, 19, 9653.

[15] K. Katagiri, Y. Tanaka, K. Uemura, K. Inumaru, T. Seki, Y. Takeoka NPG Asia Materials, 2017, 9, e355. 
[16] Y. Takeoka, S. Yoshioka, A. Takano, S. Arai, N. Khanin, H. Nishihara, M. Teshima, Y. Ohtsuka, T. Seki, Angew. Chem. Int. Ed. , 2013, 52, 7261.

[17] S.F. Liew, J. Forster, H. Noh, C.F. Schreck, V. Saranathan, X.Lu, L; Yang, R.O. Prum, C.S. O’Hern, E.R. Dufresne, H. Cao, Optics express, 2011, 19, 9, 8208.

[18] I.A. Menon, P.K. Basu, S. Persad, M. Avaria, C.C. Felix, B. Kalyanaraman, Br. J. Ophtalmol., 1987, 71, 7, 549.

[19] L. Simonot, A. Zobelli, M. Elias, J. Salomon, J.C. Dran, J. Trace and Microprobe Techn., 2003, 21, 1, 35.

[20] E. Checroun, A. Goyer, A. Bensalah-Ledoux, D. Carole, C. Le Luyer, A. Pillonnet, ARSAG Support Tracé, 2018, $17,82$.

[21] A. Pillonnet, A. Goyer, FR Patent 1851 1727, 2018.

[22] I. Merdrignac, D. Espinat, Oil \& Gas Science and Technology - Rev. IFP, 2007, 62, 1, 7.

[23] I.Walzack, Degree Thesis, Université de Lyon, France, 2000.

[24] C.I. Bothe, in Artists'Pigments : A Handbook of Their History and Characteristics, (ed. B.H. Berrie) Vol.4, National Gallery of Art, Washington, 2007, p.111.

[25] J. Connan, le bitume dans l'antiquité, (Ed. Errance, Paris), 2012

[26] M. Dimova, G. Panczer, M. Gaft, Annales géologiques de la péninsule balkanique, 2006, 67, 101

[27] Oil and Colour chemists'Association, in Inorganic pigments in surface coating, Vol. 1, (Springer ed., Australia,1983), Ch. 25.

[28] F. Perego, Dictionnaire des matériaux du peintre, (Ed. Belin, Paris), 2007.

[29] M. Elias, J. Lafait, La couleur, Lumière, vision et matériaux, (Ed. Belin, Paris), 2006 [30] S. Longo, M. Mauro, C. Daniel, P. Musto, G. Guerra, Carbon, 2014, 77, 896.

[31] S. Ohsawa, T. Kawamura, N. Takamatsu, Y. Yusa, J. Volcanol. Geotherm. Res., 2002, 113, 49.

[32] K.Ehlers, R. Chakrabarty, H. Moosmüller, Appl. Optics, 2014, 53, 9, 1808.

[33] P.K. Raghuprasad, Physics Essays, 2017, 30, 1, 116.

[34] H. N. Lazzarelli, Gemstones Identification, (Ed. Gembluechart), 2010, p. 4 


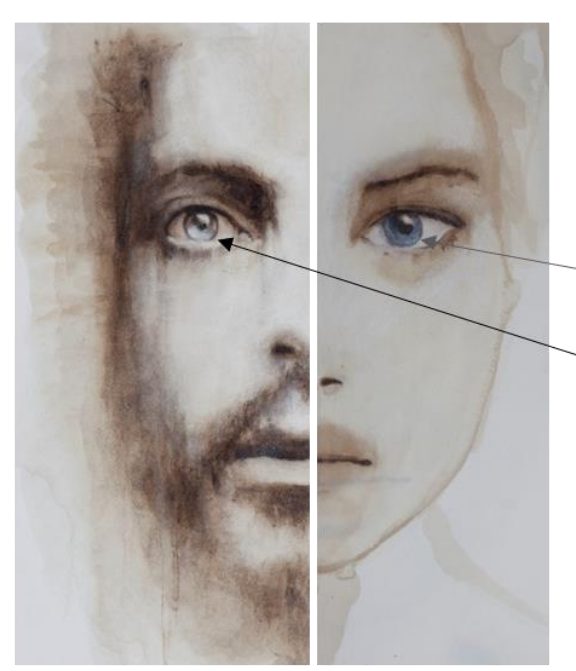

A

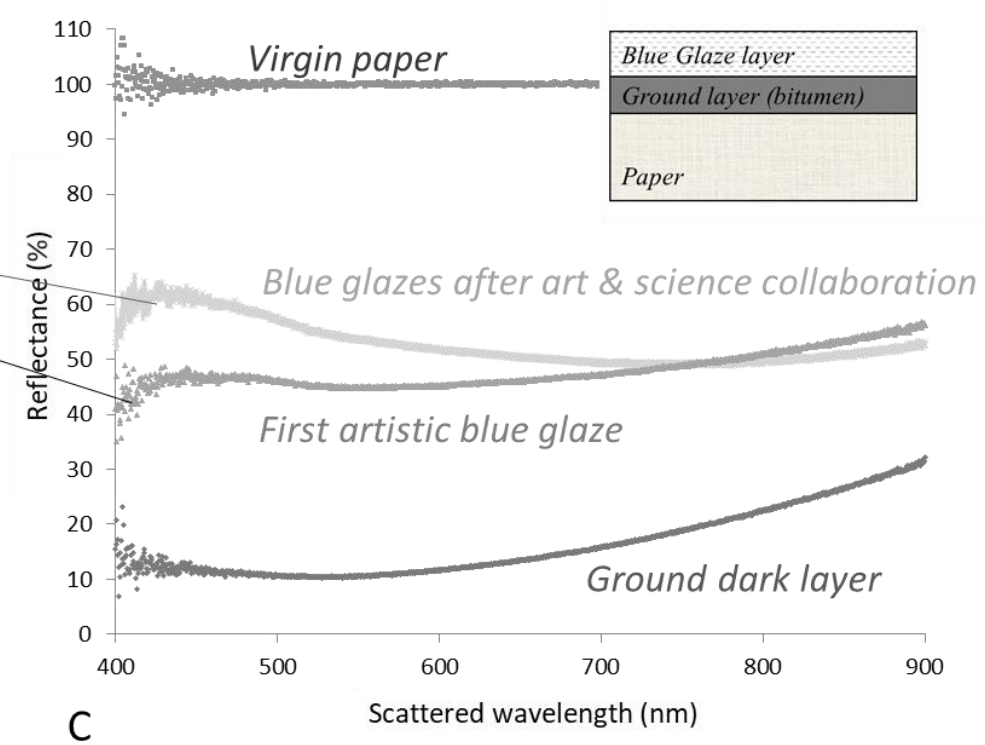

Figure 1. The eyes have been painted using an oil-based glaze using $(A)$ the artist's intuitive approach and (B) the more systematic approach based on science. (C) Reflectances of the different components normalized to virgin paper. The inset provides a schematic of the multilayer structure that is added to the paper. The blue glaze layer consists of mineral particles embedded in a transparent matrix.

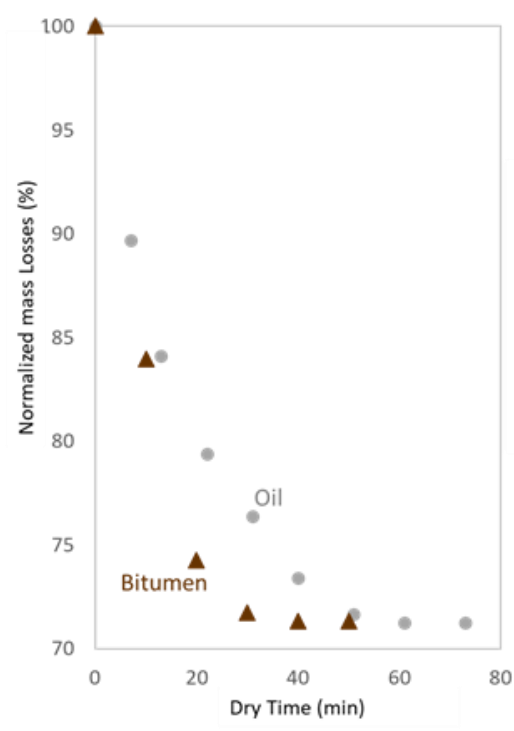

A

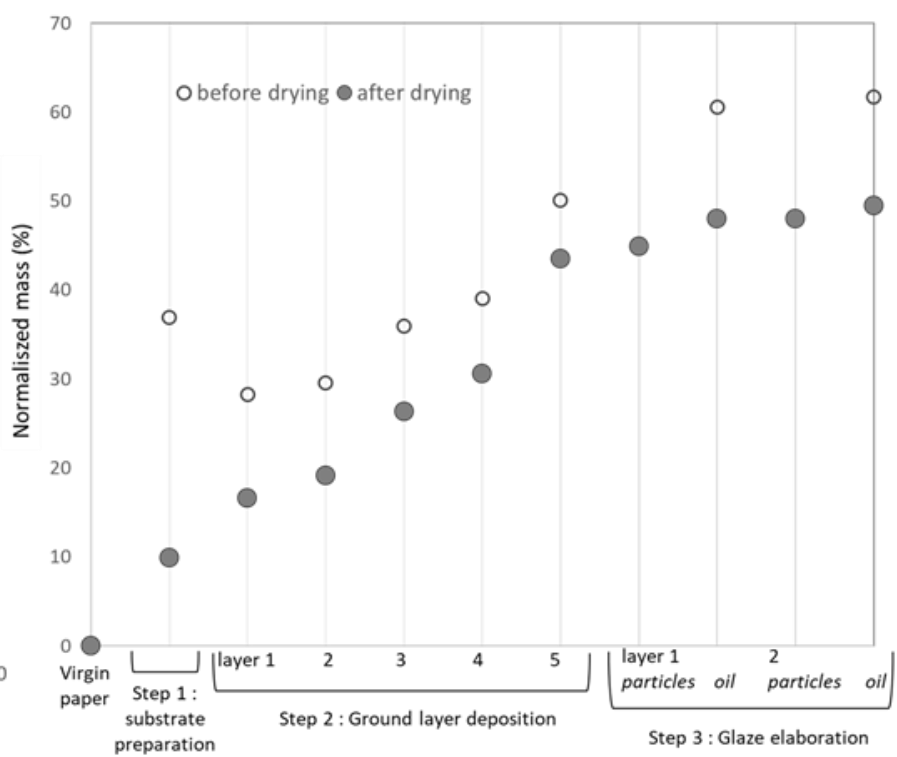

B

Figure 2. (A) Normalised mass loss after deposition of oil and bitumen on paper. $100 \%$ corresponds to the initial mass after deposition. (B) Evolution of sample weight after soaking the paper with varnish (Step 1), after adding four layers of bitumen diluted in rectified turpentine and one layer of pure bitumen (Step 2), and after adding 2 layers of dry mineral particles spray-coated with oil (Step 3). The sample weights are normalized to the weight of virgin paper used for the analysis. 
A Reflectance of raw materials

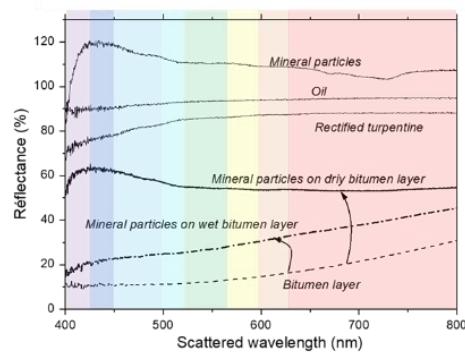

D Drawing photographs

In the sunlight

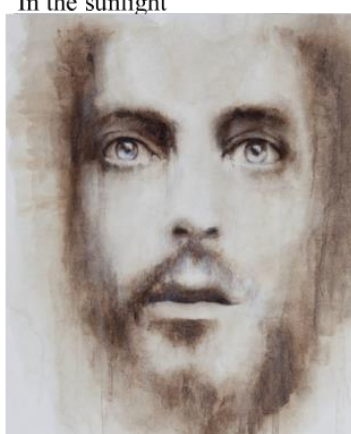

B Luminescence of mineral particles

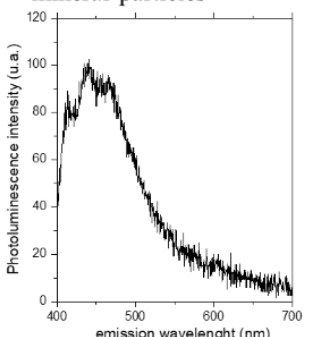

Under 354nm UV excitation

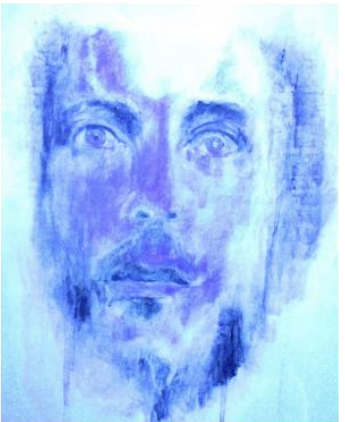

C Size distribution of mineral particles
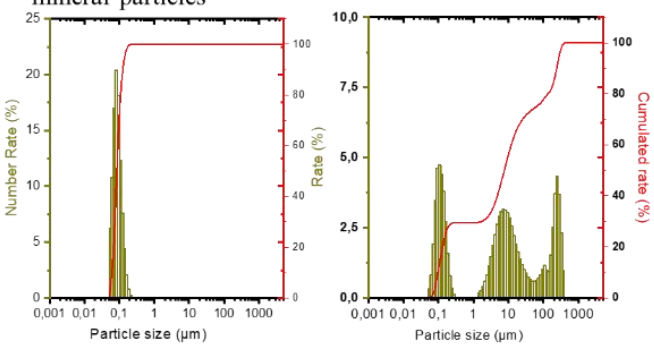

E Scheme of light matter interactions

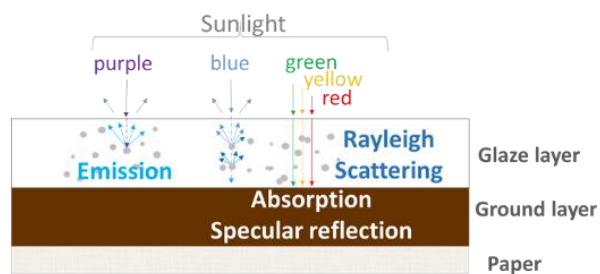

Figure 3. (A) Reflectance of raw materials deposited on virgin paper (mineral particles, varnish, rectified turpentine, and bitumen) and of mineral particles deposited on wet bitumen or dry bitumen to show the impact of the drying processes. (B) Photoluminescence of mineral particles under a 350nm excitation irradiance. (C) Size distribution of lithopone mineral particles represented in percentages of their total numbers and volume. (D) Photographs of the same painting (cf. Fig. 1A) taken under natural sunlight and under UV light at 354nm to observe the luminescence of the mineral lithopone particles. (E) Schematic of the different optical phenomena generated by the layered structure: emission by particles under UV light,

Rayleigh scattering, light absorption and reflection by the bitumen background layer.

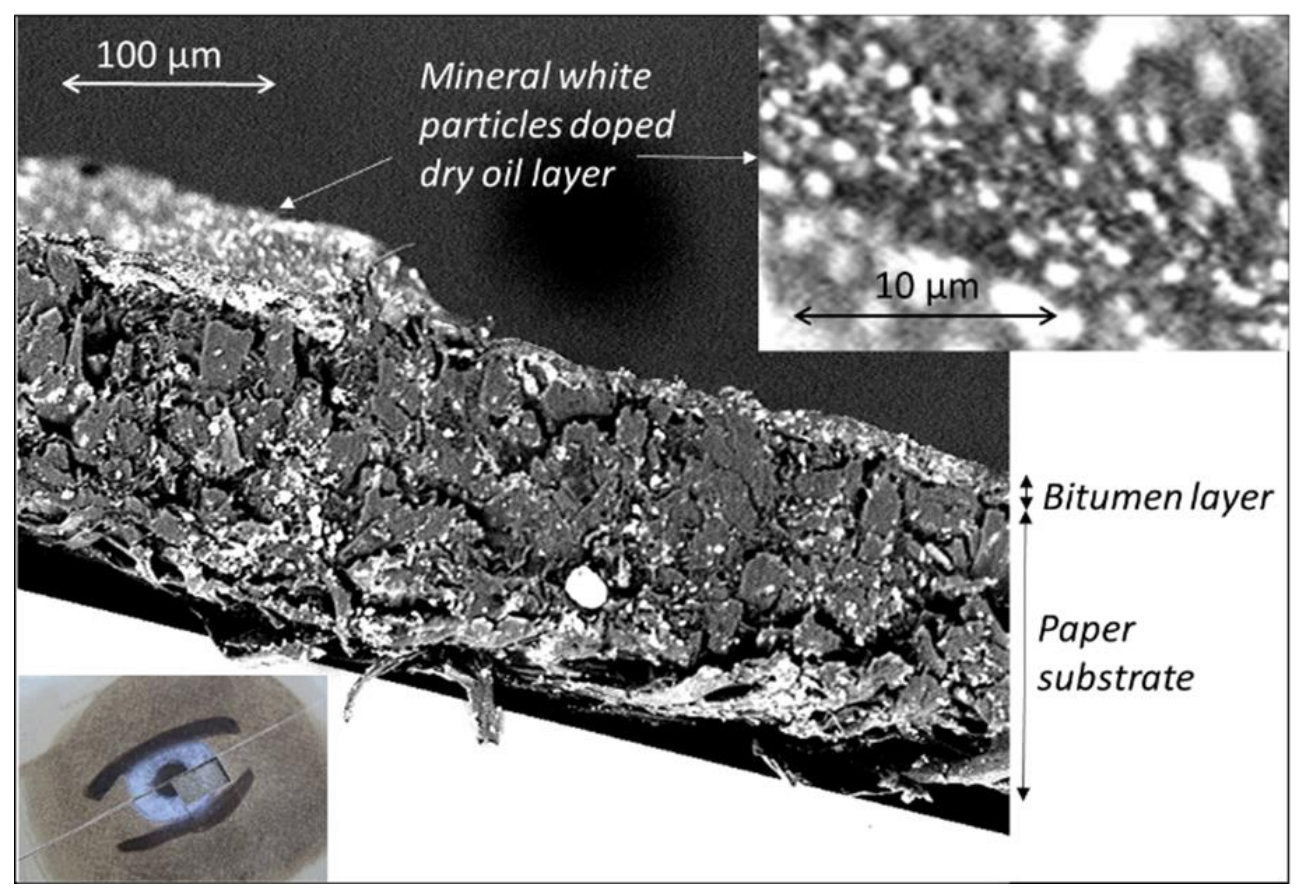

Figure 4. SEM image showing a cross section of the multilayers consisting of paper, the dark background layer of bitumen, and the top-most blue glaze layer. The inset at the top shows a magnification of the glaze layer to visualize the mineral particle distribution. At the bottom left, the analyzed sample is presented. 


\section{Supporting Information}

\section{Materials and Methods}

Pictorial sample: The paintings used in this research have been specifically elaborated for this study and do not represent the final versions of the works.

Optical reflectance measurements: Reflectance spectra were measured on an Oceanoptics USB2000 spectrometer with a measurable wavelength range between 350 and $850 \mathrm{~nm}$ (full width at half maximum of $1.5 \mathrm{~nm}$ ). The diameter of the aperture was $3 \mathrm{~mm}$. All reflectance spectra were normalized by the reflectance of white virgin paper.

Photoluminescence: The photoluminescence spectra were obtained using a 450W xenon lamp in combination with a Gemini 180 Jobin Yvon monochromator as the excitation source. The excitation light is transported to the sample by an optical fibre. The photoluminescence signal was collected via an optical fibre coupled to a Triax190 Jobin Yvon spectrometer equipped with a CCD camera. The used sample consisted of pellets of the mineral particles used in the glaze.

Photography: Photographs of the paintings were taken under natural sunlight or under $354 \mathrm{~nm}$ ultraviolet light with a Canon EOS 750D camera equipped with a Canon EFS 18-55mm lens.

X-Ray diffraction: The X-ray diffraction pattern was obtained using the D2 Phaser (Bruker, USA) with a copper anticathode in a $\theta / \theta$ configuration. Mineral particles were ground before analysis and compacted on the sample folder. The phase indexation was realized according to the PDF-2 database (International Centre for Diffraction Data).

$X$-ray fluorescence: X-ray fluorescence was measured using the energy-dispersive $\mathrm{x}$-ray fluorescence spectrometer Minipal 4 (PANanalytical, Netherlands) which uses a rhodium tube. The element indexation was realized according to the PANanalytical electronic transition energies database. Mineral particles have been ground before analysis and the powder compacted into pellets.

Particle granulometry by laser diffraction spectroscopy: The granulometry of the mineral particle powders were obtained using the Horiba Partica LA-960 laser diffractometer which measures sizes between $20 \mathrm{~nm}$ and $900 \mu \mathrm{m}$. Two light sources were used: a laser diode at $650 \mathrm{~nm}$ and an electroluminescent diode (LED) at $450 \mathrm{~nm}$. The powder was dispersed in ethanol prior to the measurements. Mie theory was applied to analyse the diffraction spectra, assuming spherical particles. The refractive indices of the particles and the solvent were taken from the literature ${ }^{[34]}$

Scanning Electron Microscopy: The structural cross-sections were obtained using a Phenom G2 Pro desktop Scanning Electron Microscope. The pictorial layers on paper were cut with a cutter blade to visualize the structural cross-section. The images were obtained using a voltage of $10 \mathrm{kV}$ corresponding to a magnification factor of 430. Higher voltages or prolonged exposure to the electron beam radiation would alter the bitumen layer.

CIELAB colorimetric measurements: The CIE colorimetric ${ }^{29}$ coordinates were determined using the portable digital PCE-CSM 1 Colorimeter with an aperture of 6mm, a D65 light source, and an observation angle of $10^{\circ}$.

Bitumen properties: The bitumen from the natural hydrocarbon source of Puy de la Poix and from Judea (commercial TITAN Betume Judaico) have been analyzed following the standardised procedures of the IFP Energies nouvelles employing a range of different techniques (see ref 20 for details). The semi quantification of 24 elements have been determined using Inductively Coupled Plasma Spectroscopy. The O, N, S heteroatom content has been measured by thermic conductivity 
and infrared spectroscopy. Different techniques such as gas chromatography and High Performance Liquid Chromatography were used to identify the different generic families present in bitumen. The refractive index has been measured with a refractometer using D-line in sodium light with a wavelength of $589 \mathrm{~nm}$. The volumetric mass was measured at $15^{\circ} \mathrm{C}$ and the viscosity at $100^{\circ} \mathrm{C}$.

\section{Supplementary analyses}

Bitumen properties: The properties of bitumen sourced from Puy de la Poix were analyzed and compared to those of Judea bitumen. Percentage differences of the different components are shown in Tables S1 and S2. A classification of the different components according to their polarity is shown in Table S3. The refractive index, volume mass, and viscosity are shown in Table $\mathbf{S 4}$.

Mineral particles properties: The chemical properties of the white mineral powders (PW6 and PW5 Rembrandt soft pastel) were analyzed using X-ray diffraction (Figure S1A) and X-ray fluorescence (Figure S1B). They contain a mixture of lithopone $\left(\mathrm{ZnS}\right.$ and $\left.\mathrm{BaSO}_{4}\right)$ with small amounts of rutile $\left(\mathrm{TiO}_{2}\right)$.

CIE-L*a*b* colour measurements: Colour data were measured in CIELAB colour space ${ }^{29}$, where $\mathrm{L}^{*}$ is the lightness parameter, $\mathrm{a}^{*}$ provides the greenness or redness, and $\mathrm{b}^{*}$ is the yellowness or blueness of the sample. Lightness is measured in values from 0 (total black) to 100 (white), while $\mathrm{a} * / \mathrm{b} *$ can range from -128 (green/blue) to 127 (red/yellow).

As the colour was not uniform across the measurement areas (the pupils in the paintings, cf. Figure S2) results are given as the range covering the lightest to the darkest shades of blue (Table S3).

For comparison, the CIE-L*a*b* colour parameters of different stacked layers realized with bitumen from Puy of Poix, diluted in rectified turpentine. The table S3 from the lightest of the darkest shades shows the range of brown tints.

\begin{tabular}{|c|c|c|c|c|c|}
\hline $\begin{array}{c}\text { Element } \\
{[\% \mathbf{m} / \mathbf{m}]}\end{array}$ & $\mathbf{C}$ & $\mathbf{H}$ & $\mathbf{O}$ & $\mathbf{N}$ & $\mathbf{S}$ \\
\hline Puy of Poix & 70.8 & 9.6 & 4.3 & 0.4 & 14.3 \\
\hline Judea & 85.6 & 12.1 & 1.1 & 1.2 & 0.1 \\
\hline
\end{tabular}

Table S1. Percentage content of different elements in bitumen from the natural former hydrocarbon source of Puy de la Poix and Judea bitumen.

\begin{tabular}{|c|c|c|c|c|c|c|c|c|c|c|}
\hline $\begin{array}{c}\text { Element } \\
\text { [mg.kg-1] }\end{array}$ & Al & Ca & Fe & K & Mg & Na & P & S & Ti & Ni \\
\hline Puy of Poix & 82 & $>100$ & $>100$ & 22 & 64 & $>100$ & 28 & $>10000$ & 30 & 73.6 \\
\hline Judea & 18 & 8 & 44 & 7 & 7 & 44 & $<5$ & 1648 & 2 & 117.3 \\
\hline
\end{tabular}

Table S2. Dosing of metal elements with a content greater than $10 \mathrm{mg} \cdot \mathrm{kg}-1$ in organic phase of the bitumen from the natural former hydrocarbon source of Puy de la Poix, and Judea bitumen. 


\begin{tabular}{|c|c|c|c|c|c|}
\hline $\begin{array}{c}\text { Family } \\
{[\% \mathbf{~ m} / \mathbf{m}]}\end{array}$ & \% Asphaltene & \% Resin & $\begin{array}{c}\text { \% aromatic } \\
\text { compounds }\end{array}$ & $\begin{array}{c}\text { \% saturated } \\
\text { compounds }\end{array}$ & \% losses \\
\hline Puy of Poix & 17.3 & 33.8 & $<30.0$ & $<6.0$ & 19.5 \\
\hline Judea & 24.3 & 17 & 0.6 & 1.2 & 56.9 \\
\hline
\end{tabular}

Table S3. Mass percentage of different generic families present in bitumen from the natural former hydrocarbon source of Puy de la Poix and Judea bitumen.

\begin{tabular}{|c|c|c|c|}
\hline & $\begin{array}{c}\text { Refractive index at } \\
\mathbf{5 8 6 n m}\end{array}$ & $\begin{array}{c}\text { Volume mass at } \mathbf{1 5}^{\circ} \mathbf{C} \\
{\left[\mathbf{k g} \cdot \mathbf{m}^{-3}\right]}\end{array}$ & $\begin{array}{c}\text { Viscosity at } \mathbf{1 2 5}^{\circ} \mathbf{C} \\
{[\mathbf{m P a . s}]}\end{array}$ \\
\hline Puy of Poix & $1.5586 \pm 0.0004$ & 1060 & $350 \pm 36$ \\
\hline Judea & $1.4855 \pm 0.0004$ & 889 & - \\
\hline
\end{tabular}

Table S4. Properties of bitumen from the natural former hydrocarbon source of Puy de la Poix, and Judea bitumen.

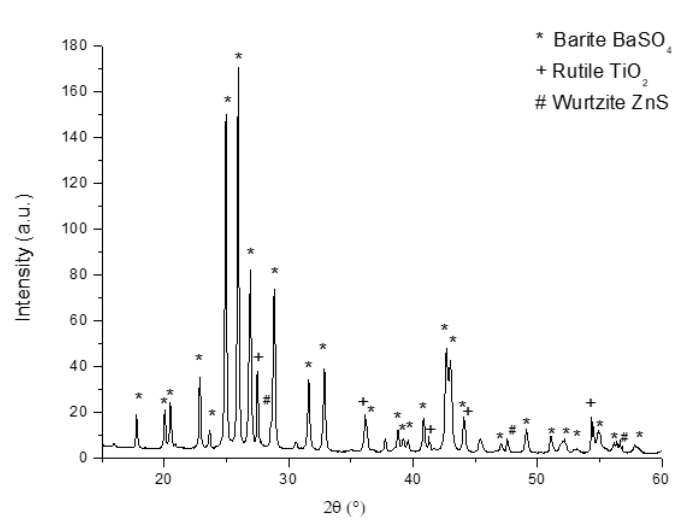

A

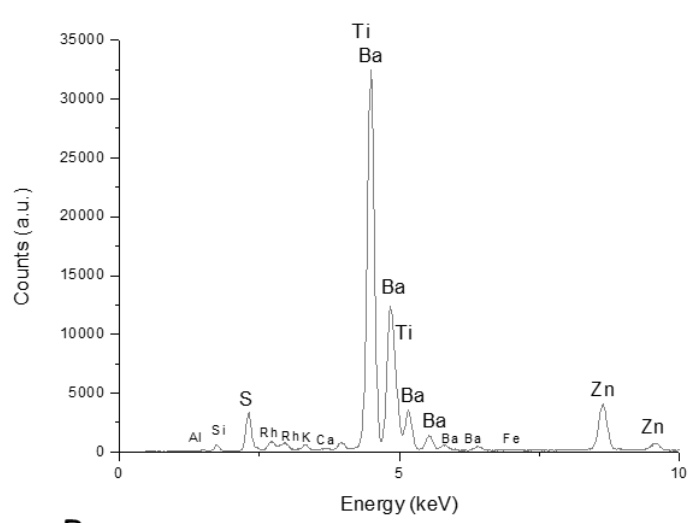

B

Figure S1. (A) X-Rays diffraction pattern showing the presence of Barite $\mathrm{BaSO}_{4}$, Rutile $\mathrm{TiO}_{2}$ and Wurtzite $Z n S$. (B) X-rays fluorescence spectra corroborating the composition.

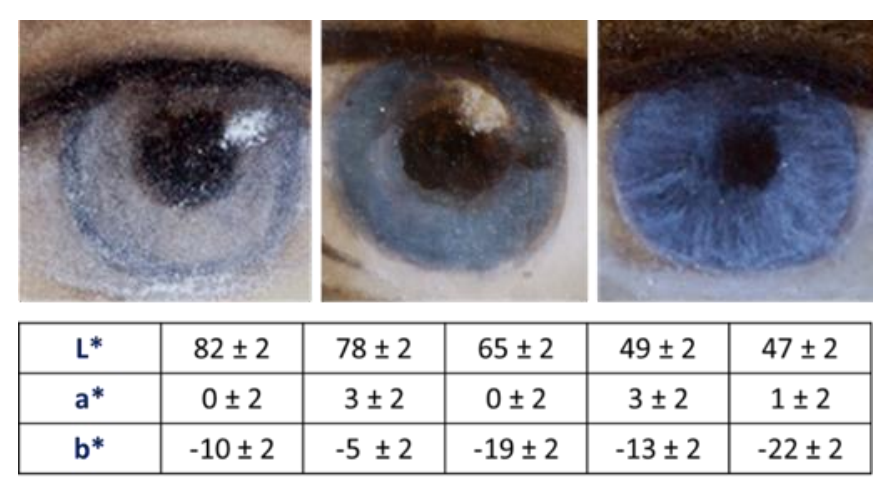

Figure 1. CIE- $L^{*} a^{*} b^{*}$ color parameters measured on different blue eyes and in different part of these blue eyes realized on paper. The table from the lightest to the darkest shades shows the range of blue tints produced by this method. 